\title{
An exploratory randomised trial of a simple, brief psychological intervention to reduce subsequent suicidal ideation and behaviour in patients admitted to hospital for self-harm
}

Christopher J. Armitage, Wirda Abdul Rahim, Richard Rowe and Rory C. O'Connor

\section{Background}

Implementation intentions link triggers for self-harm with coping skills and appear to create an automatic tendency to invoke coping responses when faced with a triggering situation.

\section{Aims}

To test the effectiveness of implementation intentions in reducing suicidal ideation and behaviour in a high-risk group.

\section{Method}

Two hundred and twenty-six patients who had self-harmed were randomised to: (a) forming implementation intentions with a 'volitional help sheet'; (b) self-generating implementation intentions without help; or (c) thinking about triggers and coping, but not forming implementation intentions. We measured self-reported suicidal ideation and behaviour, threats of suicide and likelihood of future suicide attempt at baseline and then again at the 3-month follow-up.

\section{Results}

All suicide-related outcome measures were significantly lower at follow-up among patients forming implementation intentions compared with those in the control condition (ds $>0.35$ ). The volitional help sheet resulted in fewer suicide threats $(d=0.59)$ and lowered the likelihood of future suicide attempts $(d=0.29)$ compared with patients who selfgenerated implementation intentions.

\section{Conclusions}

Implementation intention-based interventions, particularly when supported by a volitional help sheet, show promise in reducing future suicidal ideation and behaviour.

\section{Declaration of interest}

None.

\section{Copyright and usage}

(c) The Royal College of Psychiatrists 2016.
Suicidal and non-suicidal self-harm extorts significant social and economic costs. Despite the inclusion of non-suicidal self-injury and suicidal behaviour disorder in section 3 of the DSM-5 (for disorders requiring further research), there is still no international consensus on which terms best describe the wide range of selfinjurious behaviours. As self-injurious behaviour is rarely driven by a sole motive, in this paper self-harm is used to refer to all forms of self-injurious behaviour irrespective of motive(s). People admitted to hospital following an episode of self-harm are 30 times more likely to die by suicide than those in the general population, ${ }^{1}$ and even modest reductions in self-harm would bring considerable savings to healthcare services. ${ }^{2}$ Recent research has therefore focused on testing brief interventions that can be delivered to patients before they are discharged from hospital. ${ }^{3}$ These brief interventions have shown promise in reducing suicidal ideation and behaviour, but they may be limited by typically not being based explicitly on psychological theories of behaviour change and in focusing on heterogeneous patient groups. ${ }^{3}$ The aim of the present research was to test a brief theory-based psychological intervention to reduce suicidal ideation and behaviour among patients admitted following an episode of self-harm.

People engage in self-harm for many different reasons, ${ }^{4}$ but common among these reasons are triggers or critical situations (e.g. defeat, entrapment) in which people feel compelled to selfharm, ${ }^{5}$ and the implication is that providing people with the means to respond effectively to these critical situations might lessen the likelihood of an act of self-harm. Implementation intentions - tools based on Gollwitzer's ${ }^{6}$ model of action phases might be helpful in this regard because they work by automatising appropriate responses to critical situations.
Implementation intentions are 'if-then' planning that works by linking in the memory of a critical situation ('if') with an appropriate response ('then'). For the purposes of the present research, we asked participants to identify 'critical situations' in which they may have been tempted to self-harm and link them with 'appropriate responses', such as consciousness raising and stimulus control, ${ }^{7}$ that would help overcome those situations. The principal idea behind implementation intentions is that specifying the circumstances in which one will act (e.g. 'If I am tempted to self-harm when I feel trapped ... ') ensures that the appropriate response (e.g. ' . . . then I will do something else instead of self-harming') will be triggered at the appropriate time and place in the future. One key feature of implementation intentions is that they seem to operate beyond conscious awareness by enhancing the salience of the critical situation and automatising the appropriate response mechanisms that are supported by meta-analysis. ${ }^{8}$ It is also clear that the way in which these plans are formed is critical: for example, Armitage ${ }^{9}$ showed that asking people simply to form plans had no effect on subsequent alcohol consumption, whereas implementation intentions significantly reduced alcohol intake.

There is a large body of research attesting to the efficacy of implementation intention-based interventions for changing behaviour in field settings. Gollwitzer \& Sheeran ${ }^{8}$ identified 94 independent tests of implementation intentions (including laboratory tests) that yielded an average effect size of $d=0.65$. Of these 94 studies, however, none was concerned with self-harm, and no studies of the potential impact of implementation intentions on suicidal ideation and behaviour have yet been published.

Thus, the main aim of the present research was, for the first time, to test the ability of implementation intentions to reduce 
suicidal ideation and behaviour in a high-risk group. In addition, we sought to address two further issues that have arisen in the broader literature in relation to implementation intentions. First, the effects of implementation intentions on behaviour change have typically been tested among students ${ }^{8}$ and have not yet been tested among clinical populations.

Second, in field studies, people are asked to self-generate implementation intentions, i.e. participants are provided with instructions that ask them to produce both critical situations and appropriate responses and then create their own implementation intentions. Although this approach has been shown to be successful in bringing about behaviour change, it is not clear whether this method would be effective in a sample of people who have been admitted to hospital following an episode of self-harm. Thus, in addition to asking people to self-generate implementation intentions to reduce suicidal ideation and behaviour, we wanted to test a tool designed to assist implementation intention formation, namely, a volitional help sheet. ${ }^{10}$

Volitional help sheets are designed to provide a standard means by which people can form their own implementation intentions and so overcome the need for participants to selfgenerate implementation intentions. ${ }^{10}$ The volitional help sheet for self-harm provides participants with the critical situations they may encounter and the responses they might use to ensure they avoid self-harming. The content of the volitional help sheet draws on theories of suicidal behaviour, ${ }^{3}$ the self-harm motivation literature $^{11}$ and the transtheoretical model of change ${ }^{7}$ and provides a theoretically driven framework on which participants can build their own implementation intentions. To date, volitional help sheets have successfully reduced cigarette smoking ${ }^{10}$ and alcohol consumption; ${ }^{12}$ however, and consistent with the broader implementation intention literature, the volitional help sheet has not yet been tested in the domain of self-harm, nor in clinical samples.

Based on the research reviewed earlier, there are two rationales underpinning the present research. First, there is a need to reduce suicidal ideation and behaviour in a cost-effective manner. Second, although implementation intentions have been shown consistently to change behaviour, no studies have yet tested the ability of implementation intentions to improve treatment outcomes in relation to self-harm. We hypothesised that: (a) implementation intentions will significantly reduce suicidal ideation and behaviour; and (b) using a tool to support the formation of implementation intentions (a volitional help sheet ${ }^{10}$ ), as opposed to asking people to form their own (selfgenerated) implementation intentions, will maximise reductions in suicidal ideation and behaviour.

\section{Method}

\section{Participants}

Both the Kuala Lumpur Hospital and the University of Sheffield ethics committees gave approval to conduct the research. Participants were assured of confidentiality and anonymity (personal codes were used to identify individuals to preserve confidentiality and facilitate masking), and were made aware of their right to withdraw from the study or have their data removed at any point with no adverse consequences.

Two hundred and seventy-eight individuals who had been admitted to Kuala Lumpur Hospital following an episode of self-harm (ICD-10 X60-X84, intentional self-harm ${ }^{13}$ ) were approached between 1 March 2010 and 28 February 2011. We asked potential participants to read a patient information sheet and provide signed informed consent before taking part in the

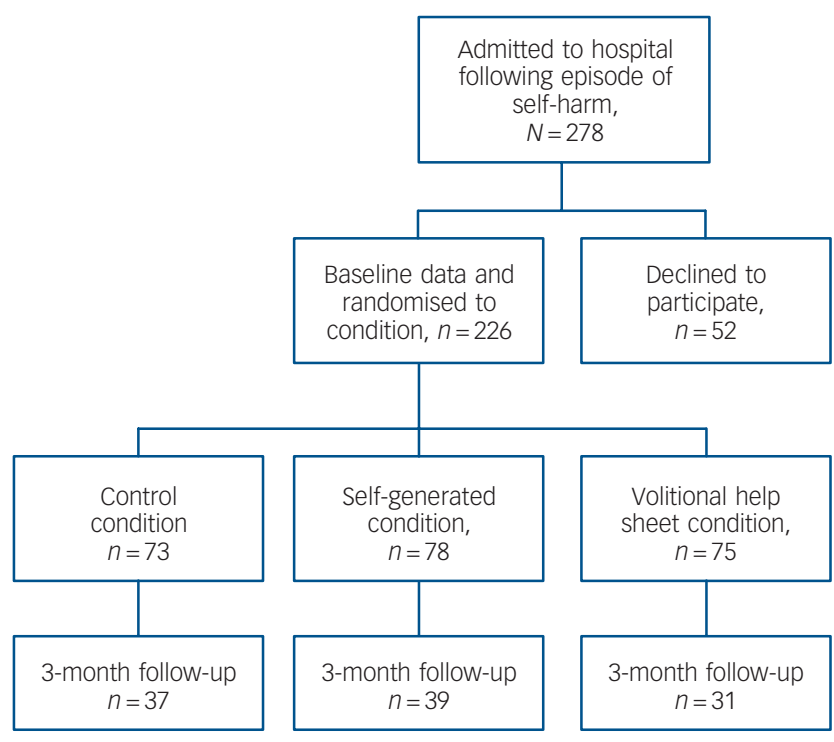

Fig. 1 Flow of participants through the study phases.

Data were analysed according to intention-to-treat with the last observation carried forward.

study (Fig. 1). Although we offered no incentive for participation, $226(81 \%)$ people initially agreed to take part in the study.

\section{Design}

We used a mixed-measures design with one between-participants factor and one within-participants factor. Condition (control $v$. self-generated implementation intention $v$. volitional help sheet implementation intention) was the between-participants factor, and time (baseline $v$. 3-month follow-up) was the within-participants variable. The main outcome measure was suicidal ideation and behaviour. The 3-month follow-up period was chosen because this is the modal time to self-harm repetition. ${ }^{14}$ We attempted to conduct an additional follow-up at 6 months. However, substantial attrition ( $>80 \%$ from baseline, $n=35$ at the 6-month follow-up) means that we lacked sufficient confidence in the reliability and validity of the findings to present them in the main text. We attribute the substantial rate of attrition to three main factors that were due to the administrative arrangements in Malaysian healthcare or a condition of gaining ethical approval: (a) postal follow-up; (b) lack of incentives; and (c) lack of reminders. Nevertheless, using identical analyses to those reported in the body of the text (i.e. standard intention-to-treat, last observation carried forward), the pattern of findings at the 6-month follow-up is identical (i.e. statistically significant differences in favour of the volitional help sheet condition) to the pattern of findings at the 3-month follow-up.

\section{Procedure}

Once informed consent was received, participants were given a baseline questionnaire to complete on their own, which was collected subsequently by a site investigator who was independent of the research team. The interventions were placed at the end of the identical-looking questionnaires, which had previously been sorted into random order using a web-based randomiser. This meant that, as far as was feasible, the site investigator was masked to the condition; research staff with knowledge of treatment 
allocation had no interactions with patients while they were in hospital or at discharge. Because Kuala Lumpur Hospital does not have an anonymised central database for self-harm events, participants were asked to provide contact details if they were willing to complete follow-up measures. One hundred and seven participants $(47 \%)$ were successfully contacted again and completed the 3-month follow-up questionnaires (Fig. 1). Baseline and follow-up questionnaires were matched using personal codes; contact details were kept separate from the data. The data were analysed according to intention-to-treat, with the last observation carried forward.

\section{Interventions}

We presented participants in all three conditions with a brief statement designed to encourage them to plan not to self-harm: 'We want you to plan not to self-harm. Research shows that you are much more likely to be successful in your intention not to self-harm if you can identify critical situations and appropriate responses'. Following this statement, we gave participants randomised to the self-generated implementation intention condition standard ${ }^{9}$ implementation intention instructions: 'You are free to choose how you will do this, but we want you to formulate your plans in as much detail as possible. Please pay particular attention to the situations in which you will implement these plans'. We left participants space in which to write their implementation intentions.

Participants in the volitional help sheet implementation intention condition had a volitional help sheet appended to their questionnaires following the brief statement encouraging them to plan to stop self-harming. The volitional help sheet was similar to those used to support implementation intention formation and successful health behaviour change in previous research. ${ }^{10}$ It consisted of a table with two columns each containing lists of 11 critical situations and 11 appropriate responses (Appendix). The 11 critical situations were derived from items used to measure self-harm triggers ${ }^{4,11,15,16}$ and the 11 appropriate responses were derived from items used to measure the processes of change outlined in Prochaska \& DiClemente's ${ }^{7}$ transtheoretical model. The critical situations tap the range of motives that typically underpin self-harm (including suicidal and non-suicidal motives). The temptation items were translated into 'if' statements, for example: 'If I am tempted to self-harm when I want to get relief from a terrible state of mind'; the processes of change items were translated into 'then' statements, for example, 'then I will think about the impact of my self-harming on the people around me'. There was one item for each of the 11 processes of change. We then asked participants in the volitional help sheet condition to draw links between as many critical situations and appropriate responses as they wanted and thereby form implementation intentions.

We also gave participants in the control condition a volitional help sheet, but did not instruct them to form implementation intentions. Instead, we simply asked them to identify critical situations and appropriate responses that might be useful to them.

\section{Measures}

\section{Suicidal ideation and behaviour}

We measured suicidal ideation and behaviour with the revised Suicidal Behaviours Questionnaire. ${ }^{17}$ The four items assess suicidal ideation and behaviour ('Have you ever thought about or attempted to kill yourself?'), frequency of suicidal thoughts ('How often have you thought about killing yourself in the past year?'), threats to die by suicide ('Have you ever told someone that you were going to die by suicide, or that you might do it?') and self-reported likelihood of suicide attempts ('How likely is it that you will attempt suicide someday?'). The suicidal ideation and behaviour items ask about suicidal thoughts, plans and attempts with and without a wish to die. At follow-up, we framed these questions in relation to suicidal ideation and behaviour in the preceding 3 months.

\section{Depression}

We measured depression with the Beck Depression Inventory-II, ${ }^{18}$ which had good internal reliability at baseline $(\alpha=0.75)$ and at the 3-month follow-up $(\alpha=0.75)$.

\section{Motivation}

We assessed motivation to avoid self-harming in the future by adapting standard measures of behavioural intention and selfefficacy assessed on seven-point $(+1$ to +7$)$ scales. ${ }^{10}$ We measured behavioural intention with three items (e.g. 'I intend to avoid deliberately harming myself - definitely do not/definitely do'). Internal reliability at both baseline $(\alpha=0.41)$ and 3 -month follow-up $(\alpha=0.40)$ was low. We measured self-efficacy with three items (e.g. 'My avoiding deliberately harming self is difficult/ easy'). Internal reliability was $\alpha=0.71$ at baseline and $\alpha=0.69$ at the 3-month follow-up.

\section{Data analysis}

We tested randomisation with multivariate analysis of variance (MANOVA); we tested the effects of the interventions with repeated-measures ANOVAs and analyses of covariance (ANCOVAs) that controlled for baseline values. Because this was an exploratory study, we chose not to specify a main outcome measure a priori, nor to apply Bonferroni's correction to the statistical comparisons.

\section{Results}

\section{Representativeness check}

Consistent with studies of self-harm prevalence around the world, significantly more women than men were admitted for self-harm. Consistent with Malaysian data, ${ }^{19,20}$ the Indian subgroup accounted for a significantly higher proportion of admissions than would be expected by chance (Table 1).

\section{Randomisation check}

We checked randomisation with MANOVA. The independent variable was condition with three levels: control $v$. selfgenerated implementation intention $v$. volitional help sheet implementation intention. The dependent variables were age, gender, suicidal ideation and behaviour, frequency of suicidal thoughts, threats to die by suicide, likelihood of attempting suicide again, depression, behavioural intention and self-efficacy at baseline. The MANOVA was non-significant, $F(18,430)=$ 1.23, $P=0.23, \eta_{\mathrm{p}}=0.05, d=0.46$, as were all the univariate tests, indicating success in the randomisation procedure (Table 2).

\section{Effects of the interventions}

Initially, we tested the effects of the interventions with mixed ANOVAs. Condition was the between-participants factor and time (baseline v. 3-month follow-up) was the within-participants factor. The dependent variables were suicidal ideation and 


\begin{tabular}{|c|c|c|c|}
\hline Variable & $\begin{array}{l}\text { Sample } \\
(n=226)\end{array}$ & $\begin{array}{l}\text { Population }^{\mathrm{a}} \\
(N=1674621)\end{array}$ & $\begin{array}{c}\chi^{2} \text { for difference between sample } \\
\text { and population }\end{array}$ \\
\hline Gender, \% & & & $39.16(P<0.01)$ \\
\hline Male & 30.1 & 50.9 & \\
\hline Female & 69.9 & 49.1 & \\
\hline \multicolumn{4}{|l|}{ Age, \% } \\
\hline 0-14 years & 0.0 & 22.1 & $64.11(P<0.01)$ \\
\hline $15-64$ years & 96.0 & 73.2 & $59.98(P<0.01)$ \\
\hline 65 years and older & 4.0 & 4.7 & $0.26(P=0.61)$ \\
\hline \multicolumn{4}{|l|}{ Ethnicity, ${ }^{\mathrm{b}} \%$} \\
\hline Malay & 30.1 & 45.9 & $24.17(P<0.01)$ \\
\hline Chinese & 10.6 & 43.2 & $97.76(P<0.01)$ \\
\hline Indian & 50.0 & 10.3 & $385.30(P<0.01)$ \\
\hline Others & 1.3 & 0.6 & $2.00(P=0.16)$ \\
\hline
\end{tabular}

\begin{tabular}{|c|c|c|c|c|c|c|c|c|}
\hline \multirow[b]{2}{*}{ Baseline variables } & \multicolumn{2}{|c|}{$\begin{array}{l}\text { Control } \\
(n=73)\end{array}$} & \multicolumn{2}{|c|}{$\begin{array}{l}\text { Self-generated intervention } \\
\qquad(n=78)\end{array}$} & \multicolumn{2}{|c|}{$\begin{array}{l}\text { Volitional help sheet intervention } \\
\qquad(n=75)\end{array}$} & \multirow[b]{2}{*}{$P^{\mathrm{b}}$} & \multirow[b]{2}{*}{$d^{\mathrm{b}}$} \\
\hline & Mean $^{\mathrm{a}}$ & s.d. & Mean $^{a}$ & s.d. & Mean $^{\mathrm{a}}$ & s.d. & & \\
\hline Age (years) & 28.25 & 11.44 & 30.26 & 12.34 & 31.57 & 16.07 & 0.32 & 0.20 \\
\hline Gender $($ men = 1, women = 2) & 1.71 & 0.46 & 1.63 & 0.49 & 1.76 & 0.43 & 0.20 & 0.20 \\
\hline Suicidal ideation and behaviour & 2.79 & 1.30 & 2.47 & 1.39 & 2.83 & 1.51 & 0.23 & 0.20 \\
\hline Frequency of suicidal thoughts & 1.99 & 0.72 & 1.74 & 0.75 & 1.92 & 0.78 & 0.12 & 0.29 \\
\hline Threats to die by suicide & 1.30 & 0.62 & 1.36 & 0.60 & 1.29 & 0.65 & 0.78 & 0.11 \\
\hline $\begin{array}{l}\text { Likelihood of attempting suicide } \\
\text { again }\end{array}$ & 2.42 & 2.05 & 2.13 & 1.92 & 2.69 & 2.01 & 0.22 & 0.20 \\
\hline Depression & 20.19 & 7.52 & 18.74 & 6.84 & 18.09 & 5.58 & 0.15 & 0.29 \\
\hline Behavioural intention & 3.44 & 0.52 & 3.42 & 0.65 & 3.51 & 0.54 & 0.63 & 0.13 \\
\hline Self-efficacy & 3.32 & 0.55 & 3.21 & 0.78 & 3.25 & 0.56 & 0.54 & 0.14 \\
\hline
\end{tabular}

behaviour, frequency of suicidal thoughts, threats to die by suicide, likelihood of attempting suicide again, depression, behavioural intention and self-efficacy (Table 3 ).

There were non-significant condition $\times$ time interactions for: frequency of suicidal thoughts, $F(2,223)=2.66, P=0.07, \eta_{\mathrm{p}}{ }^{2}=$ $0.02, d=0.29$; depression, $F(2,223)=1.48, P=0.23, \eta_{\mathrm{p}}{ }^{2}=0.01$, $d=0.20$; behavioural intention, $F(2,223)=1.42, \quad P=0.24$, $\eta_{\mathrm{p}}{ }^{2}=0.01, d=0.20$; and self-efficacy, $F(2,223)=2.83, P=0.06$, $\eta_{\mathrm{p}}{ }^{2}=0.02, d=0.29$ (Table 3). However, there were significant interactions between time and condition for: suicidal ideation and behaviour, $F(2,223)=4.95, P<0.01, \eta_{\mathrm{p}}{ }^{2}=0.04, d=0.41$; threats to die by suicide, $F(2,223)=6.20, P<0.01, \eta_{\mathrm{p}}{ }^{2}=0.05, d=0.46$; and likelihood of future suicide attempt, $F(2,223)=4.78, P<0.01$, $\eta_{\mathrm{p}}{ }^{2}=0.04, d=0.41$. The subsequent analyses clarified these significant interactions (Figs 2-5).

\section{Suicidal ideation and behaviour}

Repeated-measures ANOVAs, run separately for each condition, revealed significant decreases in suicidal ideation and behaviour between baseline and follow-up, $F s(1,72-77)=9.99-40.58$, $P s<0.01, \eta_{\mathrm{p}}{ }^{2} \mathrm{~s}>0.12, d \mathrm{~s}>0.74$; the largest decrease in suicidal ideation and behaviour was associated with the volitional help sheet condition, $F(2,74)=40.58, \quad P<0.01, \eta_{\mathrm{p}}{ }^{2}=0.35, d=1.47$.
Between-participants ANCOVAs controlling for suicidal ideation and behaviour at baseline showed significant differences between conditions at follow-up, $F(2,222)=8.70, P<0.01, \eta_{\mathrm{p}}{ }^{2}=0.07$, $d=0.55$. Planned simple contrasts revealed significant differences $(P s<0.01)$ between the control and both implementation intention formation conditions, but the self-generated and volitional help sheet conditions did not differ significantly from one another $(P=0.13)$.

\section{Threats to die by suicide}

Repeated-measures ANOVAs, run separately for each condition, revealed significant decreases in threats to die by suicide between baseline and the 3-month follow-up in the volitional help sheet condition, $F(1,74)=13.77, P<0.01, \eta_{\mathrm{p}}{ }^{2}=0.16, d=0.87$, but not in the control or self-generated conditions, $F s(2,72,77)=1.00$, $P s>0.30, \eta_{\mathrm{p}}{ }^{2} \mathrm{~s}<0.02, d \mathrm{~s}<0.29$. Between-participants ANCOVAs controlling for threats to die by suicide at baseline showed significant differences between conditions at follow-up, $F(2,222)=8.96$, $P<0.01, \eta_{\mathrm{p}}{ }^{2}=0.07, d=0.55$. Planned simple contrasts revealed a significant difference $(P<0.01)$ between the control and volitional help sheet conditions, but no significant difference $(P=0.70)$ between the control and self-generated conditions. The volitional help sheet and self-generated conditions differed significantly 


\begin{tabular}{|c|c|c|c|c|}
\hline \multirow[b]{2}{*}{ Variables } & \multicolumn{2}{|c|}{ Baseline } & \multicolumn{2}{|c|}{ Follow-up } \\
\hline & Mean $^{\mathrm{a}}$ & s.d. & Mean $^{a}$ & s.d. \\
\hline \multicolumn{5}{|l|}{ Suicidal ideation and behaviour } \\
\hline Control, $n=73$ & 2.79 & 1.30 & 2.44 & 1.01 \\
\hline Self-generated, $n=78$ & 2.47 & 1.39 & 1.96 & 0.99 \\
\hline Volitional help sheet, $n=75$ & 2.83 & 1.51 & 1.95 & 1.02 \\
\hline \multicolumn{5}{|l|}{ Frequency of suicidal thoughts } \\
\hline Control, $n=73$ & 1.99 & 0.72 & 2.00 & 0.73 \\
\hline Self-generated, $n=78$ & 1.74 & 0.75 & 1.77 & 0.77 \\
\hline Volitional help sheet, $n=75$ & 1.92 & 0.78 & 1.79 & 0.81 \\
\hline \multicolumn{5}{|l|}{ Threats to die by suicide } \\
\hline Control, $n=73$ & 1.30 & 0.62 & 1.26 & 0.53 \\
\hline Self-generated, $n=78$ & 1.36 & 0.60 & 1.32 & 0.52 \\
\hline Volitional help sheet, $n=75$ & 1.29 & 0.65 & 1.03 & 0.66 \\
\hline \multicolumn{5}{|c|}{ Likelihood of future suicide attempt } \\
\hline Control, $n=73$ & 2.42 & 2.05 & 2.16 & 1.82 \\
\hline Self-generated, $n=78$ & 2.13 & 1.92 & 1.87 & 1.60 \\
\hline Volitional help sheet, $n=75$ & 2.69 & 2.01 & 1.92 & 1.57 \\
\hline \multicolumn{5}{|l|}{ Depression } \\
\hline Control, $n=73$ & 20.19 & 7.52 & 17.78 & 6.51 \\
\hline Self-generated, $n=78$ & 18.74 & 6.84 & 16.20 & 6.56 \\
\hline Volitional help sheet, $n=75$ & 18.09 & 5.58 & 16.65 & 5.92 \\
\hline \multicolumn{5}{|l|}{ Behavioural intention } \\
\hline Control, $n=73$ & 3.44 & 0.52 & 3.56 & 0.54 \\
\hline Self-generated, $n=78$ & 3.42 & 0.65 & 3.58 & 0.68 \\
\hline Volitional help sheet, $n=75$ & 3.51 & 0.54 & 3.51 & 0.62 \\
\hline \multicolumn{5}{|l|}{ Self-efficacy } \\
\hline Control, $n=73$ & 3.32 & 0.55 & 3.60 & 0.55 \\
\hline Self-generated, $n=78$ & 3.21 & 0.78 & 3.57 & 0.58 \\
\hline Volitional help sheet, $n=75$ & 3.25 & 0.56 & 3.44 & 0.55 \\
\hline
\end{tabular}

from one another $(P<0.01, d=0.59)$, meaning participants in the volitional help sheet condition made significantly fewer threats to die by suicide at follow-up.

Likelihood of future suicide attempt

Repeated-measures ANOVAs, run separately for each condition, revealed significant decreases in likelihood of future suicide

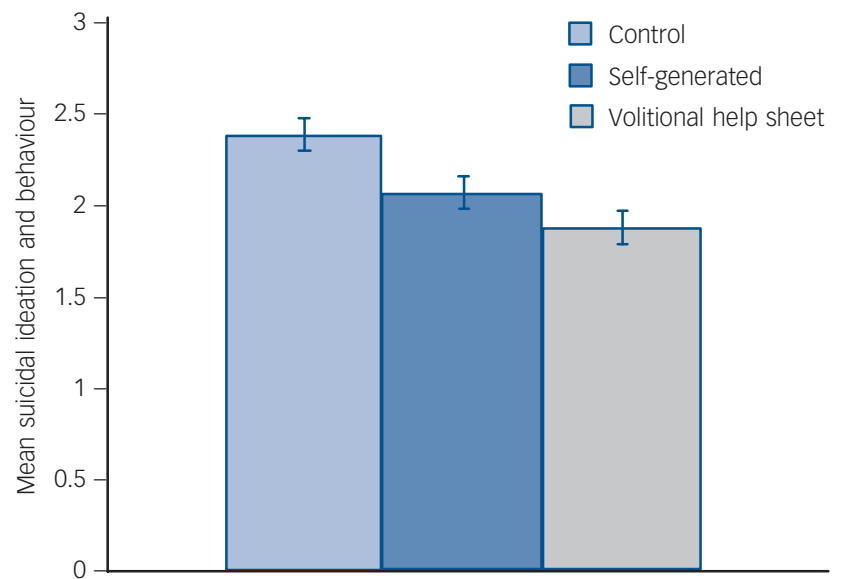

Condition

Fig. 2 Effects of the interventions on the main suicide-related outcomes at 3-month follow-up (adjusted for baseline): suicidal ideation and behaviour.

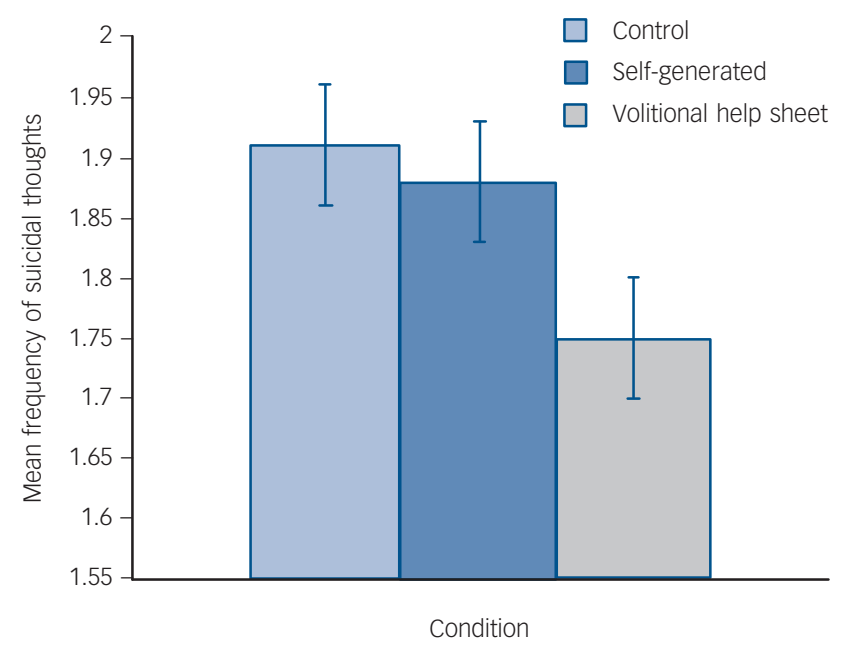

Fig. 3 Effects of the interventions on the main suicide-related outcomes at 3-month follow-up (adjusted for baseline): frequency of suicidal thoughts.

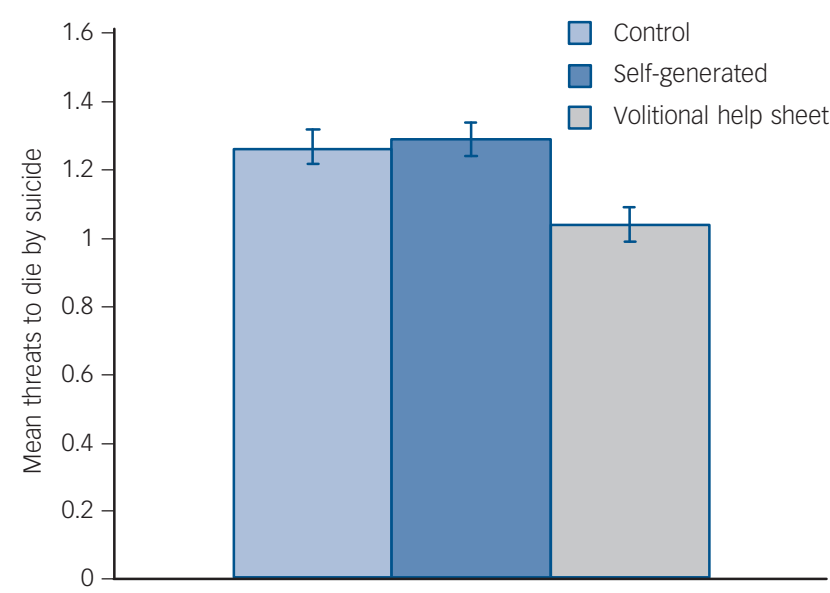

Condition

Fig. 4 Effects of the interventions on the main suicide-related outcomes at 3-month follow-up (adjusted for baseline): threats to die by suicide.

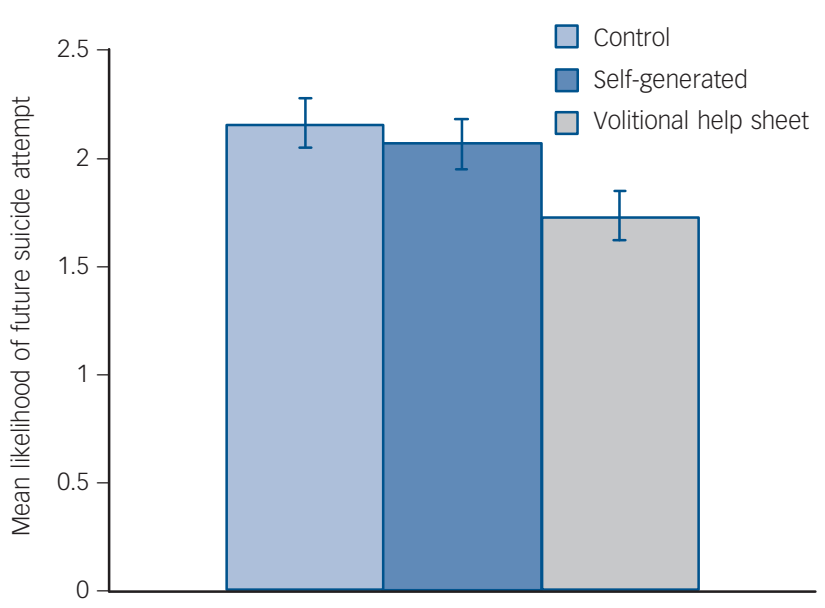

Condition

Fig. 5 Effects of the interventions on the main suicide-related outcomes at 3-month follow-up (adjusted for baseline): likelihood of future suicide. 
attempt between baseline and follow-up across all conditions, $F \mathrm{~s}(1, \quad 72-77)=4.25-23.52, \quad P \mathrm{~s}<0.05, \quad \eta_{\mathrm{p}}{ }^{2} \mathrm{~s}>0.05, \quad d \mathrm{~s}>0.46$, although the largest decrease in likelihood of future suicide attempt was associated with the volitional help sheet condition, $F(1,74)=$ 23.52, $P<0.01, \eta_{\mathrm{p}}{ }^{2}=0.24, d=1.12$. Between-participants ANCOVAs controlling for the likelihood of future suicide attempt at baseline showed significant differences between conditions at follow-up, $F(2,222)=3.81, P=0.02, \eta_{\mathrm{p}}{ }^{2}=0.03, d=0.35$. Planned simple contrasts revealed a significant difference $(P<0.01)$ between the control and the volitional help sheet conditions, but not between the control and self-generated conditions $(P=0.56)$. The selfgenerated and volitional help sheet conditions also differed significantly from one another at follow-up $(P=0.04, d=0.29)$ with people in the volitional help sheet condition reporting significantly lower likelihood of a future suicide attempt controlling for baseline.

\section{Discussion}

This is the first study to use implementation intentions to try to reduce suicidal ideation and behaviour among patients admitted to hospital following an episode of self-harm. The principal finding was that implementation intention formation was associated with lower levels of suicidal ideation and behaviour, fewer threats to die by suicide and lower reported likelihood of a future suicide attempt at follow-up. The effects were more pronounced when implementation intention formation was structured by use of a supporting tool, the volitional help sheet, which significantly augmented the effects of implementation intention formation on threats to die by suicide, and reported likelihood of a future suicide attempt. Moreover, because we employed an 'active' control group, we were able to control for people's exposure to the critical situations and appropriate responses described in the volitional help sheet. This means that explicit implementation intention formation is necessary for behaviour change to occur. The following discussion focuses on the theoretical and practical implications of the findings.

To date, implementation intention research has been characterised by student samples and short follow-up periods. ${ }^{8}$ The present research extends the evidence base to a clinical sample with a 3-month follow-up. This is important because one implication of the present research is that some samples may need support in implementation intention formation and that volitional help sheet represents one way in which this might be achieved.

Consistent with a large body of research on the impact of implementation intention-based interventions on behaviour change, the present study showed that implementation intentions were effective in reducing suicidal ideation and behaviour in a clinical setting without potentially costly health professional time, tailoring or targeting. Together, these findings suggest that the volitional help sheet potentially represents a unique, non-invasive, low-cost tool that can be used to prevent repetition of suicidal ideation and behaviour. However, the question arises as to whether the effects could be enhanced with further input from a health professional. For example, Luszczynska et al ${ }^{21}$ showed that, in a study of women enrolled in a commercial weight loss programme, participants who were asked to form implementation intentions with the help of feedback lost significantly more weight than women in the control group. Thus, the beneficial effects of implementation intention formation may be augmented by interaction with a health professional and could boost the effects observed in the present study. Indeed, as implementation intention-based interventions are not focused on the causes of an individual's distress, such interventions are likely to prove most useful when employed as an adjunct to other forms of psychosocial or pharmacological treatments.

Consistent with previous research, ${ }^{10}$ the impact of implementation intentions on suicidal ideation and behaviour was not mediated by behavioural intention or self-efficacy, which provides further support for the claim that implementation intentions represent a case of strategic automaticity whereby they operate immediately, efficiently and beyond conscious awareness. ${ }^{6}$ It would be valuable to develop measures that could tap these constructs in field settings so that the mechanism by which implementation intentions operate in the field could be verified. Nevertheless, there is considerable laboratory research showing the suggested mechanisms. ${ }^{8}$

Although the present research makes contributions both to the literature on suicidal ideation and behaviour and to the implementation intention literature, it is important to highlight some potential limitations. First, the sample was heterogeneous, including all self-harm presentations irrespective of whether the participants presented with suicidal or non-suicidal self-harm. This is not problematic for the administration of the help sheet per se as the volitional help sheet was designed for use in all cases of self-harm, irrespective of motivation. However, as a consequence of heterogeneity, it is not clear whether the effects are equally applicable to individuals who present to hospital with suicidal and non-suicidal behaviour or with people with different suicidal histories. Second, the outcome measures, although selected for their brevity and established psychometric properties, did not distinguish clearly between suicidal thoughts and behaviours. For example, one of the items was: 'Have you ever thought about or attempted to kill yourself?'

A third limitation is that, for practical reasons, the follow-up was restricted to mailed materials, which meant that attrition was inevitable and that the outcome measures had to be self-reported. Although attrition was handled using standard intention-to-treat analyses and made no difference to the principal findings, it would be valuable in the future to devote more resources to ensure that participants complete the study. Fourth, it would be useful to have a more objective outcome measure, such as future hospital admissions, although this is not currently possible in the Malaysian context. Fifth, as far as was feasible, the person collecting the data was masked to condition, but in future research it would be valuable to probe explicitly the success or failure of such concealment procedures. Sixth, because this was an exploratory study, we chose not to specify a main outcome measure a priori, nor to apply Bonferroni's correction to the statistical comparisons. In future definitive trials, we should be able to generate more precise estimates of effect size and hence specify the main outcome measure in advance.

Last, the volitional help sheet shows promise as a brief, costeffective tool to reduce suicidal ideation and behaviour, threats to die by suicide and reported likelihood of a future suicide attempt. Further research is required to replicate the findings with a more complete data-set and objective outcome measures.

\footnotetext{
Christopher J. Armitage, PhD, Manchester Centre for Health Psychology, School of Psychological Sciences, Manchester Academic Health Science Centre, University of Manchester; Wirda Abdul Rahim, PhD, Richard Rowe, PhD, Department of Of Manchester; Wirda Abdul Rahim, PhD, Richard Rowe, PhD, Department of
Psychology, University of Sheffield; Rory C. O'Connor, PhD, Institute of Health and Wellbeing, University of Glasgow, UK

Correspondence: Chris Armitage, Manchester Centre for Health Psychology, School of Psychological Sciences, Manchester Academic Health Science Centre University of Manchester, Coupland Street, Oxford Road, Manchester M13 9PL, UK. Email: chris.armitage@manchester.ac.uk

First received 2 Apr 2014, final revision 18 Dec 2014, accepted 26 Feb 2015
} 


\section{Appendix: Self-harm volitional help sheet (intervention instructions)}

We want you to plan to avoid self-harming. Research shows that if people can spot situations in which they will be tempted to self-harm and then link them with a way to overcome those situations, they are much more likely to be successful in avoiding self-harming.

On the left-hand side of the table is a list of common situations in which people feel tempted to self-harm; on the right-hand side of the table is a list of possible solutions.

For each situation that applies to you personally (left-hand side), please draw a line linking it to a solution (right-hand side) that you think might work for you. Please draw a line linking one situation to one solution at a time, but make as many (or as few) situation-solution links as you like.

\begin{tabular}{|c|c|}
\hline $\begin{array}{l}\text { Situations } \\
\square \text { If I am tempted to self-harm when I want to get relief from a terrible } \\
\text { state of mind }\end{array}$ & $\begin{array}{l}\text { Solutions } \\
\square \text { Then I will do something else instead of self-harming }\end{array}$ \\
\hline$\square$ If I am tempted to self-harm when I want to punish myself & $\square$ Then I will tell myself that I can stop self-harming if I want to \\
\hline$\square$ If I am tempted to self-harm when I want to die & $\begin{array}{l}\text { Then I will recall information people have given me about the benefits } \\
\text { of stopping self-harming }\end{array}$ \\
\hline $\begin{array}{l}\square \text { If I am tempted to self-harm when I want to show how desperate } \\
\text { I am feeling }\end{array}$ & $\begin{array}{l}\square \text { Then I will tell myself that society is changing in ways that make it easier } \\
\text { for people to stop self-harming }\end{array}$ \\
\hline $\begin{array}{l}\square \text { If I am tempted to self-harm when I want to find out whether } \\
\text { someone really loves me }\end{array}$ & $\square$ Then I will make sure I am rewarded by others if I don't self-harm \\
\hline$\square$ If I am tempted to self-harm when I want to get some attention & $\begin{array}{l}\square \text { Then I will think about the impact of my self-harming on the people } \\
\text { around me }\end{array}$ \\
\hline$\square$ If I am tempted to self-harm when I want to frighten someone & $\begin{array}{l}\square \text { Then I will remember that I react emotionally to warnings about my } \\
\text { self-harming }\end{array}$ \\
\hline $\begin{array}{l}\square \text { If I am tempted to self-harm when I want to get my own back } \\
\text { on someone }\end{array}$ & $\begin{array}{l}\square \text { Then I will remember that I get upset when I think about my } \\
\text { self-harming }\end{array}$ \\
\hline$\square$ If I am tempted to self-harm when I feel defeated & $\begin{array}{l}\square \text { Then I will put things around my home or place of work that remind } \\
\text { me not to self-harm }\end{array}$ \\
\hline$\square$ If I am tempted to self-harm when I feel trapped & $\begin{array}{l}\square \text { Then I will seek out someone who listens when I need to talk about } \\
\text { self-harm }\end{array}$ \\
\hline$\square$ If I am tempted to self-harm when I feel hopeless & $\square$ Then I will take medication \\
\hline
\end{tabular}

\section{References}

1 Cooper J, Kapur N, Webb R, Lawlor M, Guthrie E, Mackway-Jones K, et al. Suicide after deliberate self-harm: a 4-year cohort study. Am J Psychiatry 2005; 162: 297-303

2 Sinclair JM, Gray A, Rivero-Arias O, Saunders KE, Hawton K. Healthcare and social services resource use and costs of self-harm patients. Soc Psychiatry Psychiatr Epidemiol 2011; 46: 263-71.

3 O'Connor RC, Rasmussen S, Beautrais A. Recognition of suicide risk, crisis helplines, and psychosocial interventions: a selective review. In Internationa Handbook of Suicide Prevention: Research, Policy and Practice (eds RC O'Connor, S Platt, J Gordon): 435-56. Wiley-Blackwell, 2011.

4 Hjelmeland $\mathrm{H}$, Hawton $\mathrm{K}$, Nordvik $\mathrm{H}$, Bille-Brahe U, De Leo D, Fekete S, et al. Why people engage in parasuicide: a cross-cultural study of intentions. Suicide Life Threat Behav 2002; 32: 380-93.

5 O'Connor RC, Smyth R, Ferguson E, Ryan C, Williams JM. Psychological processes and repeat suicidal behavior: a four-year prospective study. J Consult Clin Psychol 2013; 81: 1137-43.

6 Gollwitzer PM. Implementation intentions: strong effects of simple plans. Am Psychol 1999; 54: 493-503.

7 Prochaska JO, DiClemente CC. Stages and processes of self-change of smoking: toward an integrative model of change. J Consult Clin Psychol 1983: 51: 390-5

8 Gollwitzer PM, Sheeran P. Implementation intentions and goal achievement: a meta-analysis of effects and processes. Adv Exp Soc Psychol 2006; 38 : 69-119.

9 Armitage CJ. Effectiveness of experimenter-provided and self-generated implementation intentions to reduce alcohol consumption in a sample of the general population: a randomized exploratory trial. Health Psychol 2009; 28 $545-53$
10 Armitage CJ. A volitional help sheet to encourage smoking cessation: a randomized exploratory trial. Health Psychol 2008; 27: 557-66.

11 Madge N, Hewitt A, Hawton K, de Wilde EJ, Corcoran P, Fekete S, et al. Deliberate self-harm within an international community sample of young people: comparative findings from the Child \& Adolescent Self-harm in Europe (CASE) study. J Child Psychol Psychiatry 2008; 49: 667-77.

12 Armitage CJ, Arden MA. A volitional help sheet to reduce alcohol consumption in the general population: a field experiment. Prev Sci 2012; 13: $635-43$.

13 World Health Organization. ICD-10 Version: 2010. WHO, 2011 (http:// apps.who.int/classifications/icd10/browse/2010/en/X64)

14 Kapur N, Gask L. Introduction to suicide and self-harm. Psychiatry 2009; 8 233-6.

15 O'Connor RC. The integrated motivational-volitional model of suicidal behavior. Crisis 2011; 32: 295-8.

16 Williams JMG. Cry of Pain: Understanding Suicide and Self-harm. Penguin Books, 1997.

17 Osman A, Bagge CL, Gutierrez PM, Konick LC, Kopper BA, Barrios FX. The Suicidal Behaviors Questionnaire-Revised (SBQ-R): validation with clinical and nonclinical samples. Assessment 2001; 8: 443-54.

18 Beck AT, Steer RA, Brown GK. Manual for the Beck Depression Inventory-II. Psychological Corporation, 1996.

19 Armitage CJ, Abdul Rahim W, Rowe R, O'Connor RC. Trends in self-harm in Kuala Lumpur, 2005-2011. Arch Suicide Res, in press.

20 Armitage CJ, Panagioti M, Abdul Rahim W, Rowe R, O'Connor RC. Completed suicides and self-harm in Malaysia: a systematic review. Gen Hosp Psychiatry 2015; 37: 153-65

21 Luszczynska A, Sobczyk A, Abraham C. Planning to lose weight: randomized controlled trial of an implementation intention prompt to enhance weight reduction among overweight and obese women. Health Psychol 2007; 26: $507-12$ 